\title{
A STUDY OF CORRELATION OF PAP SMEAR AND COLOPOSCOPY WITH HISTOPATHOLOGICAL FINDINGS IN CASES OF UNHEALTHY CERVIX
}

KEY WORDS: Colposcopy, Pap Smear, Histopathology, Cervical malignancy, HSIL, LSIL

\section{Dr S. Lavanya}

(M.D),

\section{Dr S. Sakthim}

adhubala*
(M.B.B.S), *Corresponding Author

\section{Dr Gali Niroosha (M.D),}

BACKGROUND: Cervical malignancy is the second most common among the malignancies in females, with breast carcinoma the first. Screening of unhealthy cervix by pap smear and supported by colposcopy and histopathology can easily identify the premalignant lesions and also other non-invasive inflammatory lesions of the cervix.

OBJECTIVES: To correlate Pap smear and Colposcopy findings with histopathological findings in detecting premalignant lesions in the unhealthy cervix.

MATERIALS AND METHODS: A prospective observational study was conducted on 100 symptomatic, sexually active women of 20-60 years. Pap smears and colposcopy was done for all 100 women who came with complaints of white discharge per vagina, intermenstrual, postcoital, or postmenopausal bleeding. The final correlation of the Pap smear and colposcopy was based on histopathology reports.

RESULTS: In the present study, the sensitivity of Pap smear for detecting lesions above LSIL was $82.3 \%$, specificity 96.9\%, PPV was $93.3 \%$, negative predictive value $91.4 \%$ and accuracy of Pap smear was $92 \%$. The sensitivity of colposcopy in the detection of low-grade lesions and above came out to be $94.1 \%$, specificity $87.8 \%$, positive predictive value $80 \%$, negative predictive value $96 \%$ and accuracy of colposcopy was $90 \%$.

CONCLUSION: This study suggests that the accuracy of detection of cervical abnormalities is higher when cytology, colposcopic guided cervical biopsy and colposcopy are used complimentarily in the diagnosis of cervical lesions. Colposcopy abolishes the need for repeated follow-up as in Pap smear and helps to reduce false-negative cases.

\section{INTRODUCTION}

Cancer of cervix is one of the most common gynecological malignancies in women and a significant cause of mortality in women worldwide. ${ }^{1}$ Cancer cervix has been considered preventable because it has a prolonged pre-invasive state and the availability of screening programs and treatment of pre-invasive lesion is effective. An unhealthy cervix is a group of cervical lesions, mostly chronic, which include chronic cervicitis, endocervicitis, cervical erosions lacerations, polyps and leukoplakia.

These lesions can harbor premalignant lesions even when a pap smear is negative. The gold standard of cervical screening is a pap smear, but the screening algorithm recommends Colposcopy and Colposcopic-directed cervix biopsy. The commonly received reports are inflammatory smear, LSIL, HSIL.

The aim of the study was to correlate the findings of Pap smear and Colposcopy with histopathological findings in detecting premalignant lesions of the cervix.

\section{MATERIALS AND METHODS:}

This prospective observational study was conducted on Women attending Gynecology OPD at Narayana Medical College and Hospital, Nellore. One hundred cases that fulfilled the selection criteria and gave consent for colposcopy and cervical biopsy were recruited for the study.

\section{Inclusion Criteria}

1. Age group 20 to 60 years.

2. Recurrent episodes of white discharge per vagina, Inter menstrual bleeding, post-coital bleeding, or postmenopausal bleeding.

3. Suspicious looking cervix (leukoplakia, cervical erosion, bleed on touch ulcer, etc.).

4. Women with PAP smear showing dysplasia.

\section{Exclusion Criteria}

1. Pregnant women.

2. Clinically visible growth on the cervix.

3. Prior hysterectomy or procedure on cervix.

\section{Unmarried.}

Written consent were taken from all the patients after a brief explanation of the procedure. A careful history including data like age, socioeconomic status, education, parity, age at marriage of the patient was taken. General examination and systemic examination were done.

Prepared Pap smear slides were fixed in 95\% ethyl alcohol and ether. All the women were subjected to colposcopy and cervical biopsy. Biopsy specimens were obtained in $10 \%$ formalin fixative. The prepared Pap smears slides were then stained according to the conventional Pap technique and examined under a light microscope. The cytological interpretation of the smears was reported according to the Bethesda system.

Later 5\% acetic acid was applied to the cervix and colposcopic evaluation was done under 40x magnification, colposcopicdirected biopsy was taken and processed, histopathological slides prepared and stained with hematoxylin and eosin and examined under a light microscope. Biopsy results were sorted as Cervicitis/ metaplasia, CIN - 1 (mild dysplasia/ correlating with LSIL), CIN - 2/3 (moderate to severe dysplasia/ correlating with HSIL) and Squamous cell carcinoma.

\section{Statistical Analysis}

The statistical analysis was made by calculating the diagnostic efficacy of each test. The sensitivity, specificity, positive predictive value (PPV), negative predictive value (NPV), false-positive rate, false-negative rate, and accuracy for Pap smear and Colposcopy were calculated using Colposcopy-directed biopsy results as the gold standard.

\section{RESULTS:}

In the present study, women attending the gynecology outpatient department who had Pap smears were subjected to colposcopy and colposcopy-directed biopsy. The results of histopathology were compared and analyzed.

Table 1: Distribution of cases according to Age

\begin{tabular}{|l|l|}
\hline Age group & Number of cases (n=100) \\
\hline $20-30$ yrs & 14 \\
\hline
\end{tabular}




\begin{tabular}{|l|l|}
\hline $31-40$ yrs & 44 \\
\hline $41-50$ yrs & 32 \\
\hline $50-60$ yrs & 10 \\
\hline Total & 100 \\
\hline
\end{tabular}

In the present study, many cases were in the age group of 3140 years ( $44 \%)$, followed by $41-50$ years( $32 \%)$. The mean age was 37.1 years. In the studies by Vijay Y.Kalyankar et al., ${ }^{2}$ T.S Savitha et al., ${ }^{3}$ and Krishna Algotar et al., ${ }^{4}$ M.Coppleson et al., ${ }^{5}$ most of the cases are seen in the age group of 31 to 40 years.

\section{Graph 1: Distribution of cases based on parity}

The majority of the study group were Para $2(33 \%)$ and Para 3 $(37 \%)$. In a study by Vijay Y. Kalyankar et al., ${ }^{2}$ maximum cases are seen in Para $2(45 \%)$ and above (45\%). In the study of Kriti Bhatnagar et al., ${ }^{6}$ maximum women were having parity $3-4$ $(51.2 \%)$.

\section{Table 2: Distribution of cases based on symptoms}

\begin{tabular}{|l|l|}
\hline Symptoms & Number of cases (n=100) \\
\hline White discharge & 78 \\
\hline Post-coital bleeding & 8 \\
\hline Intermenstrual bleeding & 6 \\
\hline Postmenopausal bleeding & 8 \\
\hline
\end{tabular}

The most typical symptom was recurrent white discharge per vagina $(79 \%)$ in the present study. In Kriti Bhatnagar's study ${ }^{6}$, the white discharge was seen in $66.4 \%$ of cases and in T.S.Savitha's study ${ }^{3}$, it was $86 \%$ of cases.

\section{Table 3: Pap smear results}

\begin{tabular}{|l|l|}
\hline PAP Smear & Number of cases \\
\hline NILM & 6 \\
\hline Inflammatory & 64 \\
\hline LSIL & 14 \\
\hline HSIL & 10 \\
\hline AGC & 2 \\
\hline SCC & 4 \\
\hline Total & 100 \\
\hline
\end{tabular}

The specificity and sensitivity of Pap smear in our study were $96.9 \%$ and $82.3 \%$, respectively. The specificity and sensitivity of pap smear were $95 \%$ and $81 \%$ in the study by Smitha Krishna Gowda et al ${ }^{7}$.

The positive predictive value was $93.3 \%$ for pap smears in the present study. The negative predictive value was $91.4 \%$ for pap smears in the present study.

\section{Table 4: Colposcopic diagnosis}

\begin{tabular}{|l|l|}
\hline Colposcopic diagnosis & Number \\
\hline Normal & 6 \\
\hline Inflammatory & 54 \\
\hline CIN1 & 24 \\
\hline CIN 2 & 10 \\
\hline CIN3 & 2 \\
\hline Invasive carcinoma & 4 \\
\hline
\end{tabular}

The specificity and sensitivity of Colposcopy in our study were $87.8 \%$ and $94.1 \%$ respectively. The specificity and sensitivity of Colposcopy were $91 \%$ and $94 \%$ in the study by Smitha Krishna Gowda et $\mathrm{al}^{7}$.

The positive predictive value of the Colposcopy in the present study is $80 \%$. The negative predictive value of the Colposcopy in the present study was $96 \%$.

\section{Table 5: Histopathological results}

\begin{tabular}{|l|l|}
\hline Histopathological diagnosis & Number \\
\hline Chronic cervicitis & 66 \\
\hline CIN 1 & 18 \\
\hline CIN 2 & 10 \\
\hline CIN 3 & 2 \\
\hline
\end{tabular}

\section{SCC}

\section{4}

All 100 cases were subjected to colposcopic-directed biopsy. The majority of cases, $66 \%$, had chronic cervicitis. The positive biopsy includes 34 cases out of 100. The Biopsies were considered positive if they revealed CIN I and above. It contains 18 CIN I case, 10 cases of CIN II, 2 cases of CIN III, and 4 cases of SCC

The accuracy of Pap smear to Biopsy in the present study was $92 \%$. Similar results of accuracy were seen in the study by Smitha Krishna Gowda et al $^{7}(92 \%)$.

The accuracy of Colposcopy to Biopsy in the present study was $90 \%$. Other studies have shown colposcopic accuracy around $80 \%$ to $85 \%$.

\section{DISCUSSION:}

Cervical cancer screening is an important part of preventive health care for women ${ }^{8}$. A significant correlation is seen between pap smear and cervical histopathology.

In Total, 100 cases were selected with different cervical lesions for the study.

Table 6: Correlation between Pap smear and Biopsy

\begin{tabular}{|l|l|l|l|l|l|l|}
\hline Pap smear & \multirow{2}{*}{ No } & & Biopsy & & \\
\cline { 3 - 7 } & & $\begin{array}{l}\text { CHRONIC } \\
\text { CERVICITIS }\end{array}$ & CIN I & CIN II & CIN III & SCC \\
\hline NILM & 6 & 6 & - & - & - & - \\
\hline Inflammatory & 64 & 58 & 6 & - & - & - \\
\hline LSIL & 14 & 2 & 12 & - & - & - \\
\hline HSIL & 10 & - & - & 10 & - & - \\
\hline AGC & 2 & - & - & - & 2 & - \\
\hline SCC & 4 & - & - & - & - & 4 \\
\hline Total & 100 & 66 & 18 & 10 & 2 & 4 \\
\hline
\end{tabular}

Table 7: Correlation between Colposcopy and Biopsy

\begin{tabular}{|c|c|c|c|c|c|c|}
\hline \multirow[t]{2}{*}{ Colposcopy } & \multirow[t]{2}{*}{ Number } & \multicolumn{5}{|c|}{ BIOPSY } \\
\hline & & $\begin{array}{c}\text { Chronic } \\
\text { cervicitis }\end{array}$ & $\begin{array}{c}\text { CIN } \\
\text { I }\end{array}$ & $\begin{array}{c}\text { CIN } \\
\text { II }\end{array}$ & $\begin{array}{c}\text { CIN } \\
\text { III }\end{array}$ & SCC \\
\hline Normal & 6 & \begin{tabular}{|l|}
6 \\
\end{tabular} & - & - & - & - \\
\hline Inflammation & 54 & 52 & 2 & - & - & - \\
\hline CIN I & 24 & 8 & 16 & - & - & - \\
\hline CIN II & 10 & - & - & 10 & - & - \\
\hline CIN III & 2 & - & - & - & 2 & - \\
\hline $\begin{array}{c}\text { Invasive } \\
\text { carcinoma }\end{array}$ & 4 & - & - & - & - & 4 \\
\hline Total & 100 & 66 & 18 & 10 & 2 & 4 \\
\hline
\end{tabular}

In the present study, the 31-40 years of age group was the most common group. This indicates that premalignant lesions precede malignancy by a decade, and prompt identification by appropriate screening reduces the mortality and morbidity associated with cervical cancer.

Multiparous women were more common in our study, indicating that women with early sexual exposure in early life and more children are associated with more chances of abnormal findings in screening for cervical premalignant lesions. Many authors have discussed the strong influence of early coitus and multiparity on the risk of cervical cancer?

Abnormal colposcopic findings are more common in women with postcoital bleeding and post-menopausal bleeding, which indicate that they are significant risk factors for malignancy of the cervix.

Colposcope has the ability to localize and determine the extent of all lesions which will not be visible to the naked eye and can be detected in a single setting. It also helps in determining the site of biopsy, patients for conservative treatment of CIN, follow-up of intraepithelial neoplasia and 
invasive lesions.

On Histopathological examination, the majority of the cases in the present study were of chronic cervicitis and infection $(66 \%)$ followed by mild dysplasia $(18 \%)$, moderate to severe in $12 \%$ and $4 \%$ malignant lesions.

Hence the present study strongly suggests that colposcopy and colposcopic directed biopsy of the cervix should be included along with the Pap smear in the screening of cases for early detection of cervical cancer and its usage increases the accuracy of detection of cervical abnormalities when used complementarily.

\section{CONCLUSIONS}

Cancer cervix is one of the preventable and highly curable diseases when diagnosed in the early stages. CIN lesions and early invasive cancer are asymptomatic. So, it is essential to develop an accurate screening tool to detect the disease early when it is amenable to treatment.

In developing countries like India, Pap smear cytology, being a less expensive procedure, is used to screen cervical cancer. Findings in this study showed that Colposcopy is more sensitive than the Papanicolaou smear as a screening tool for CIN. But the Pap smear was, however, more specific as a screening tool for CIN than Colposcopy. Colposcopy by magnifying 40 times helps in determining the appropriate site of a cervical biopsy.

Hence, the accuracy of detecting cervical abnormalities is higher when cytology, Colposcopy, and colposcopic-directed cervical biopsy are used complementarily in the evaluation of women with an unhealthy cervix.

Colposcopy and cytology are not competitive methods but complementary to each other. The combined use of cytology and colposcopic-directed biopsy could result in early detection of preinvasive carcinomas.

\section{REFERENCES}

1. Maheshwari A, Kumar N, Mahantshetty U. Gynecological cancers: A summary of published Indian data. South Asian J Cancer 2016;5:1 12-20.

2. Vijay VY et al. Colposcopic evaluation of unhealthy cervix and its correlation with Papanicolau smear in cervical cancer screening. International Journal of Reproduction, Contraception and Gynaecology.2017 Nov;6(1 1)4959-4965.

3. Savitha TS, Sapna W. A comparison of pap smear, colposcopy and colposcopy directed biopsy in evaluation of unhealthy cervix. Journal of Evolution of Medical and Dental Sciences. 2015 Mar 2;4(21):3639-48.

4. Algotar K, Nalawade A, Sachdev S. Predictive value of colposcopy in cervical cancer screening. Bombay Hosp J. 2004 Jul;4603:1-9.

5. Coppleson M. The diagnosis and treatment of early (preclinical) invasive cervical cancer. Surgical management of advanced and recurrent cervical carcinoma. Clinics in Obst and Gynaecology. March 1985;149-68.

6. Bhatnagar K, Baghel K. Epidemiology of patients with bad cervix attending gynaecology OPD at a tertiary care centre in western uttar Pradesh, India. Int J Reprod Contracept Obstet Gynecol 2017;6:791-6.

7. Krishnegowda S, Veena MS. Efficacy of colposcopy technique with Pap smear and histology in screening of cervical lesions. Int J Reprod Contracept Obstet Gynecol.2014;3(3):696-702

8. Kessler TA. Cervical cancer: prevention and early detection. InSeminars in oncology nursing 2017 May l (Vol.33,No.2,pp. 172-183).WB Saunders.

9. Brinton La, Reeves Wc, Brenes Mm, Herrero R, De Brilton Rc, Gaitan E, Tenorio F, Garcia M, Rawls We. Parity As A Risk Factor For Cervical Cancer. American Journal Of Epidemiology. 1989 Sep 1;130(3):486-96. 